\title{
An Analytical Study of Power Line Effect on UTP Cable using Lumped Circuit Components
}

\author{
Mitamoni Sarma \\ Department of Electronics, LCB College, Maligoan, Guwahati, India \\ E-mail:mtsarma5805@gmail.com \\ Prof. Shikhar Kr. Sarma \\ Department of Information Technology, Gauhati University, Assam, India \\ E-mail:sks001@gmail.com
}

\begin{abstract}
The paper defines the term electrical noise with its types. Electromagnetic Interference (EMI), which is one type of electrical noise, is also defined and general techniques used for controlling EMI are described. Networking cables are affected by the EMI effect caused by a nearby power cable and data transmission through Unshielded Twisted Pair (UTP) cable, which is the mostly effected cable by EMI, may be degraded for it. Today, UTP cable is the most popular networking cable supporting 10G Ethernet. The most common effective methods for reduction of EMI effect on UTP cable, physical separation and use of shielding are described. EMI is caused by coupling mechanisms between source of interference and receptor. The two types of couplings are capacitive coupling and inductive coupling. The paper analyses and models the two couplings using lumped circuit components and electric circuit analysis considering power cable as the source of interference and networking cable as the receptor circuit of EMI.
\end{abstract}

Index Terms - Electromagnetic Interference (EMI), Physical Separation, Shielding, Mutual Inductance, Lumped Circuit Components, Capacitive Coupling, Inductive Coupling

\section{Introduction}

Noise is defined as unexpected electrical signals, which interfere or distort with an original signal and Interference is the undesirable effect of noise. When noise is generated from within the system itself, it is called internal noise and when generated from an outside source, it is called external noise ${ }^{[1, p p ~ 102,103]}$.

Internal noises are mainly categorized into two types:

i) Thermal noise (due to electron movement within the electrical circuits) and

ii) Imperfections (in the electrical design).

External noises are mainly categorized into four types: i) Natural origins (electrostatic interference and electrical storms),

ii) Electromagnetic Interference (EMI) - from currents in cables,

iii) Radio frequency interference (RFI) - from radio systems radiating signals, iv) Cross- talk (from other cables separated by a small distance).

\section{Electromagnetic Interference}

Electromagnetic Interference (EMI) is one type of external noise. It is emitted by electrical and electronic systems carrying time varying signals and which causes unwanted signals (interference or noise) to be induced in other systems. This may interrupts, obstructs resulting in degradation or limitation in the effective performance of those other systems. When cables are in close proximity of an electromagnetic field, undesired voltage and current may be induced on it due to coupling mechanisms between the two, which can interfere with the transmission of data, voice and video applications on the cable.

Electromagnetic Interference (EMI), which is an undesirable phenomenon, degrades the performance of a system due to the electromagnetic fields making up the electromagnetic environment. Electromagnetic environment is defined as the totality of electromagnetic phenomenon present at a given location [2] Electromagnetic fields produced from power cables and transformers can affect the transmissions of a signal cable.

\section{General Techniques used for Controlling EMI}

EMI can be controlled or suppressed by employing some techniques in the design process. Generally, there are three common techniques. They are: Grounding, Shielding and Filtering ${ }^{[2]}$. Each technique has a distinct role in the system design. But proper grounding may sometimes minimize the need for shielding and filtering and proper shielding may minimize the need for filtering. 
1) Grounding------Ground is a point, which is kept at a zero potential and grounding is connecting electrical and electronic equipment by a conductor to ground. The ground point is considered as the reference point for all signals in associated circuitry and any undesired current can be transferred to this point for the elimination of its effects. Again, bonding provides a low impedance path between two metal surfaces which avoids the voltage build up between the metallic parts, thus avoiding EMI.

2) Shielding------The function of a Shield is to prevent unwanted electromagnetic energy from entering to a specific region. Shields are available in different forms such as partitions or boxes as well as in the form of cable and connector shields.

3) Filtering------The function of a filter is to offer low impedance to certain frequencies while blocking the other frequencies. An electric filter can be designed using resistors, inductors and capacitors. Filters can effectively reduce EMI.

\section{Sources of EMI}

There are a variety of possible EMI sources (or noise sources) existing in the electromagnetic environment. Effect from these EMI sources can degrade the performance of an electronic system operating in their vicinity. Noise can be broadly classified into two broad categories based on their origin: natural noise and man-

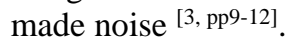

\section{Classification of Natural EMI Sources}

\section{Terrestrial Noise}

1. Atmospheric noise (noise from lighting around the world)

2. Nearby and medium distant lighting

3. Electrostatic Discharge (ESD)

\section{Extraterrestrial noise}

1. Cosmic/galactic radio noise

2. Solar noise (whistles, solar-disturbed and quite radio noise)

\section{Classification of Man-made EMI Sources}

EMI Sources due to the Power Network and its Equipment

1. Switching operations

2. Power faults

3. Electric motors

4. Static and rotary connectors

5. Rectifiers

6. Contractors
EMI sources due to industrial and commercial equipment

1. Arc furnaces

2. Induction furnaces

3. Air conditioning

4. Fluorescent lamps

5. Neon displaces

6. Medical equipment

EMI sources due to machines and tools

1. Workshop machines

2. Rolling mills

3. Cotton mills

4. Welders

5. Rotary saws

6. Compressors

7. Ultrasonic cleaners

\section{EMI sources due to communication systems}

1. Radio broadcast stations

2. TV stations

3. Radar

4. Mobile phones

5. Remote control

6. Door-opening transmitters

EMI sources due to consumer devices

1. Microwave ovens

2. Refrigerators

3. Mixers

4. Washing machines

5. Vacuum cleaners

6. Hair dryers

7. Shavers

8. Light dimmers

9. Personal computers

\section{Types of EMI Errors}

When the effect of the undesirable electromagnetic interference alters the content or context of an analog or a digital signal, the signal is said to be corrupted. A corrupted signal is a signal with its original content and error. There are two most common errors created by the effect of the undesirable EMI. They are Bit Corruption and Junction Rectification ${ }^{[2]}$. 
1) Bit Corruption------Bit Corruption occurs in digital systems. It alters the 'byte' of data in a digital system. If the altered and corrupted data bytes are interpreted as instructions, the misinterpreted instruction may cause the instrument to unexpectedly change states or attempting to perform an invalid operation. Misinterpretation of data may yield inaccurate results.

2) Junction Rectification------Junction Rectification occurs in analog equipments. It occurs when a semiconductor device is exposed or comes to the close proximity of device having a high frequency alternating current field. This exposure may cause an unintentional $\mathrm{AC}$ to DC rectification within the semiconductor junctions resulting in a direct current. These rectified signals may produce a DC offset voltage or modulated signal, which may 'interpreted' as invalid data in the device.

The remaining part of the paper is organized as follows: Section 2 gives an idea about the techniques used to protect Cabling Systems from AC Power lines. Section 3 describes briefly how and why interference occurs between power line and communication line. Electromagnetic Interference is caused by coupling mechanisms between source of interference and receptor circuits. Section 4 analyses and models different couplings mechanisms using lumped circuit components and electric circuit analysis considering power cable as the source of interference and networking cable as the receptor circuit of EMI. It also includes a brief description about the lumped circuit components. Conclusion of the paper is given in the last section of the paper.

\section{Techniques used to Protect Cabling Systems from AC Power Lines}

Today, we are surrounded by electrical wires everywhere and so an electromagnetic environment exists all around us all the times. Therefore, the performance of a network cabling system is depended upon the intensity of the EMI surrounding the system. The most important point upon which the performance of the cabling system depends on how the system is protected against EMI.

There are some techniques, which are used to eliminate or reduce electrical noise or interference. But it is important to note that noise usually cannot be eliminated; it can only be minimized to the point where it no longer causes interference ${ }^{[4,}$ p26]. Today, UTP cable is the most preferred networking cable from the points of high speed, cost and installation. According to Siemon Company, there are two effective methods to minimize the effect of EMI on UTP cablings ${ }^{[5]}$ : Physical Separation and Use of Shielding.

\subsection{Physical Separation}

By maintaining some degree of physical separation between power lines and UTP cable, the cabling system can be protected against EMI. The Siemon Company, specify the minimum distance recommended for 100 ohm UTP cabling, as well as the pathways and spaces used to carry or house the telecommunications cabling. By applying the proper physical separation distances, UTP cabling system can be used by avoiding EMI. The Siemon Company recommends UTP cabling as the preferred cabling media, in situations where it is possible to maintain a proper physical separation between power lines and UTP cabling.

\subsection{Shielding}

When minimum separation distances cannot be maintained for UTP cabling, shielding is the technique employed to protect cabling system from EMI. In shielding, noise voltage is not induced onto the conductors, it is induced into a foil or braid surrounding the twisted pairs. Shielded cabling system gives about $20 \mathrm{~dB}$ better performance than UTP cabling system in terms of interference immunity with proper installation. In designing and installing shielded cabling systems, proper grounding and bonding are very important for the effectiveness of shielded systems. One important point to note that the shielding must be complete, that means shield coverage must exist throughout the cable and its connecting hardware. The Siemon Company also recommends the minimum power separation for shielded cabling systems, which is less than UTP cabling systems.

\section{Interference between Power Line and Communication Line}

Generally communication lines run along the same route as the power lines. It is because the user of electrical energy also uses the communication system. The power line may produce interference with the communication lines when they exist in close proximity. The generated interference in a communication line from a power line, when they run in close proximity originates from the electromagnetic and electrostatic effects between them ${ }^{[6-11]}$. In ref ${ }^{[6, p 251]}$, it is pointed out that "if the power line is running along the same route as the communication line, there will be an interference in the communication line due to both electromagnetic and electrostatic effects." The current produced by the electromagnetic effect is superimposed on the communication signal and thereby distorting the signal. For electrostatic effect, a voltage is induced in the communication line, which may be dangerous in handling the communication system. 


\subsection{Electromagnetic Effect}

Figure 1 shows a three-phase $(a, b, c)$ power line conductor with communication line conductors ' $d$ ' and ' $e$ '. If $I_{a}, I_{b}, I_{c}$ are the three balanced currents through the power conductor then

$$
\mathrm{I}_{\mathrm{a}}+\mathrm{I}_{\mathrm{b}}+\mathrm{I}_{\mathrm{c}}=0
$$

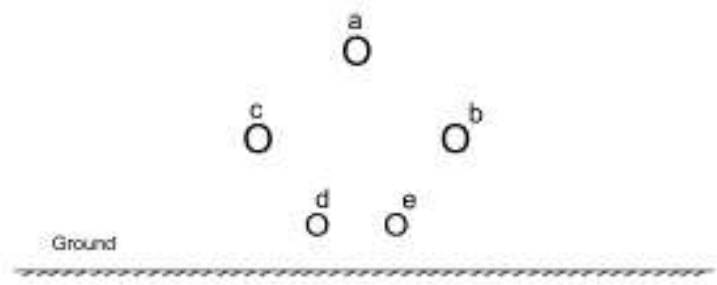

Fig. 1: Three phase line with communication line

The induced voltage in communication conductors due to electromagnetic effect is

$$
\mathrm{V}=\mathrm{j} \omega \mathrm{MI} \text { volt }
$$

Where, 'I' is the current in the power conductor and ' $\mathrm{f}$ ' is the frequency.

The value of induced voltage is directly proportional to the value of mutual inductance $M$ and current $I$. The value of $\mathrm{M}$ depends upon the distance between power line and communication line. Larger the value of the distance between the two, smaller is the value of mutual inductance.

\subsection{Electrostatic Effect}

Figure 1 is also considered for calculating the electrostatic effect on conductors ' $d$ ' and 'e'. If the potential of conductor ' $d$ ' due to charge $q$ on conductor ' $\mathrm{a}$ ' is $V_{a d}$, on the conductor ' $b$ ' is $\mathrm{V}_{\mathrm{bd}}$ and on the conductor ' $c$ ' is $\mathrm{V}_{c d}$, the total potential will be the phasor sum of all the potential as

$$
\mathrm{V}_{\mathrm{d}}=\mathrm{V}_{\mathrm{ad}}+\mathrm{V}_{\mathrm{bd}}+\mathrm{V}_{\mathrm{cd}}
$$

The potential in conductor ' $e$ ' due to electrostatic effect can also be computed.

\section{Power Effect Modeling}

\subsection{Lumped Circuit Components}

1) Resistance (R) is one of the three basic components of electric circuit theory ${ }^{[12]}$. From circuit viewpoint, resistance is expressed by ohm's law in terms of an associated current and potential difference as,

$$
\mathrm{R}=\mathrm{V} / \mathrm{I} \Omega
$$

From energy viewpoint, resistance can be expressed in terms of the dissipated energy per unit time per unit current squared. Thus,

$$
\mathrm{R}=\mathrm{W} / \mathrm{I}^{2} \mathrm{t} \Omega
$$

From geometrical viewpoint, resistance is expressed as,

$$
\mathrm{R}=\rho \mathrm{L} / \mathrm{A} \Omega
$$

Where, $\rho=$ resistivity of the material $(\Omega), \mathrm{L}=$ length of the conductor $(\mathrm{m}), \mathrm{A}=$ cross-sectional area $\left(\mathrm{m}^{2}\right)$.

2) Inductor (L) is another basic component of electric circuit theory [12]. From circuit viewpoint, an appropriate defining equation for inductance is

$$
\mathrm{L}=\mathrm{V}_{\mathrm{L}} / \mathrm{di} / \mathrm{dt} \text { volt-second/ampere or henrys }
$$

From energy viewpoint, energy is expressed as

$$
\mathrm{W}=1 / 2 \mathrm{Li}^{2} \mathrm{~J} \text { or } \mathrm{L}=2 \mathrm{~W} / \mathrm{i}^{2} \mathrm{H}
$$

Which, describes the inductance parameter in terms of the amount of energy stored in its magnetic field corresponding to its instantaneous current.

From geometrical viewpoint, inductance is expressed as,

$$
\mathrm{L}=\mathrm{N}^{2} \mu \mathrm{A}_{\mathrm{m}} / 1
$$

Where, $\mathrm{N}=$ number of turns of the inductor, $\mu=\mathrm{a}$ physical property of the magnetic material, called the permeability, $1=$ mean length of the iron core of the inductor in meters, $\mathrm{A}_{\mathrm{m}}=$ cross-sectional area of the iron.

3) Capacitance (C) is the third basic component of electrical circuit theory is the capacitor ${ }^{[12]}$. Capacitance is the proportionality factor relating the charge between two metal conductors to the corresponding potential difference existing between them. Therefore,

$$
\mathrm{q}=\mathrm{CV}_{\mathrm{C}}
$$

Where $\mathrm{q}$ is the charge and $v_{c}$ is the potential difference. is,

From circuit viewpoint, the definition of capacitance

$$
\mathrm{C}=\mathrm{i} / \mathrm{dv}_{\mathrm{c}} / \mathrm{dt} \mathrm{F}
$$

From energy viewpoint, capacitance is defined as,

$$
\mathrm{C}=2 \mathrm{~W} / \mathrm{v}_{\mathrm{c}}^{2} \mathrm{~F}
$$

From geometrical viewpoint, capacitance is defined as,

$$
\mathrm{C}=\varepsilon \mathrm{A} / \mathrm{d} \mathrm{F}
$$


Where, $\varepsilon=$ the permittivity of the material between the plates, $\mathrm{A}=$ the area of the plates, $\mathrm{d}=$ the distance between the plates of the capacitor.

\subsection{Use of Lumped Circuit Components}

For modeling the effect of coupling between two circuits, lumped circuit components are used. The condition for the use of lumped circuit components is that the physical size of the system must be smaller than the wavelength of the disturbance [13, pp52-54]. Let us consider that the length (size) of the system be ' $\mathrm{D}$ ' and the wavelength of disturbance ' $\lambda$ '. Where several wavelengths of disturbances are present, the shortest wavelength is compared with ' $D$ '. If $D<<\lambda$, the propagation time of disturbances is negligible compared to their period. In such cases, use of lumped circuit components is a good approximation to model circuit behavior.

If $\mathrm{D} \geq \lambda$, propagation effects are dominant and lumped circuit components fail to represent the phenomena accurately. In that case, the whole system is divided into small sections and the size of a section is much smaller than $\lambda$. All the sub-sections of the system can be represented by using lumped parameters separately. This technique is used only for the solution of distributed networks i.e. networks for which $\mathrm{D} \geq \lambda$.

Let us illustrate with examples the circumstances under which lumped circuit model or a distributed model is necessary. Let us consider first the study of power signal $(50 \mathrm{~Hz}$ or $60 \mathrm{~Hz})$. Say, a high voltage power line of length $\mathrm{D}=50 \mathrm{~km}$. A typical model can be used for the study of the line which is a $\Pi$ - equivalent network (fig 2).

The wavelength of a $50 \mathrm{~Hz}$ signal is,

$$
\lambda=\left(3 \times 10^{8}\right) / 50=6 \times 10^{8} \mathrm{~m}
$$

The value of ' $\lambda$ ' is much larger than the length of the line ' $\mathrm{D}$ ' i.e. $\mathrm{D} \leq \lambda$, which fulfils the conditions for lumped circuit representation.

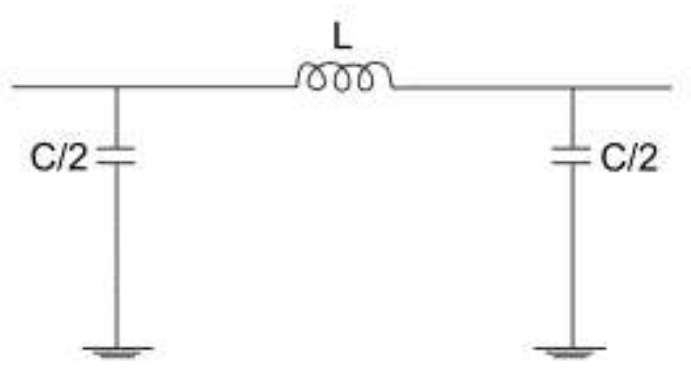

Fig. 2: A typical model of a ח- equivalent network

Again let us try to examine whether this model is suitable to study the propagation of lightning induced transients on the line. Typically, such unidirectional pulses are with a rise-time of $1 \mu \mathrm{s}$. Therefore, the associated amount of energy is with frequencies of the order of $1 \mathrm{MHz}$. The wavelength associated with $1 \mathrm{MHz}$ frequency is,

$$
\lambda=3 \times 10^{8} / 10^{6}=300 \mathrm{~m}
$$

In this case $\lambda<\mathrm{D}$, hence lumped circuit model is not suitable for the study of such unidirectional pulse.

Now, let us examine whether lumped circuit presentation is suitable or not for the study of effect of AC power line on a nearby UTP networking cable. For UTP cabling cable length runs is limited to 100 meters. Again, power line carries a $50 \mathrm{~Hz}$ signal. Therefore, the value of the wavelength of the signal is $6 \times 10^{6} \mathrm{~m}$, which is larger than the length of the cable runs. Therefore, lumped circuit model is applicable for the study of effect of AC power line on UTP cable.

\subsection{Use of Network Theory}

'Electric circuit analysis', which is an approximate analysis technique, is used during most design procedures. Electric Circuit analysis technique is based on the following assumptions:

1. All electric fields are confined to the interiors of capacitors.

2. All magnetic fields are confined to the interiors of inductors.

3. Dimensions of the circuits are small compared to the wavelength(s) under consideration.

Noise coupling channels can be represented as equivalent lumped component networks, whenever possible ${ }^{[4]}$. A time varying electric field existing between two conductors can be represented by a capacitor between the two conductors (fig 3 ).
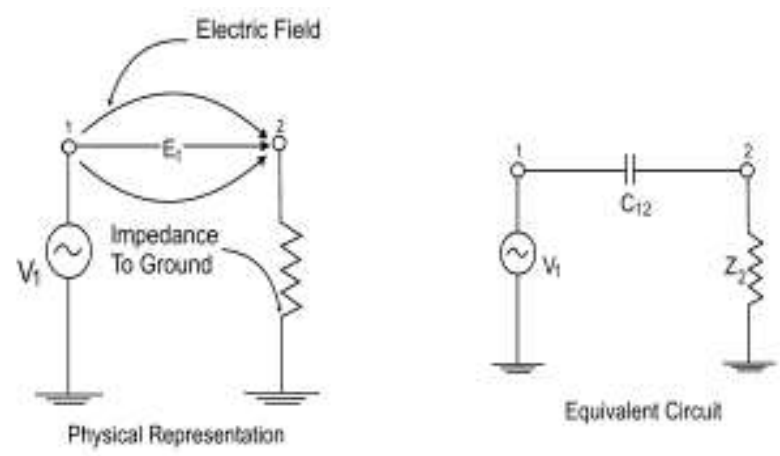

Fig. 3: When two circuits are coupled by an electric field, the coupling can be represented by a capacitor

Similarly, a time varying magnetic field coupling two conductors can be represented by the mutual inductance between the two circuits (fig 4). The condition for the validity of this type of approach is that the physical dimensions of the circuits must be small compared to the wavelengths of the signals involved. 

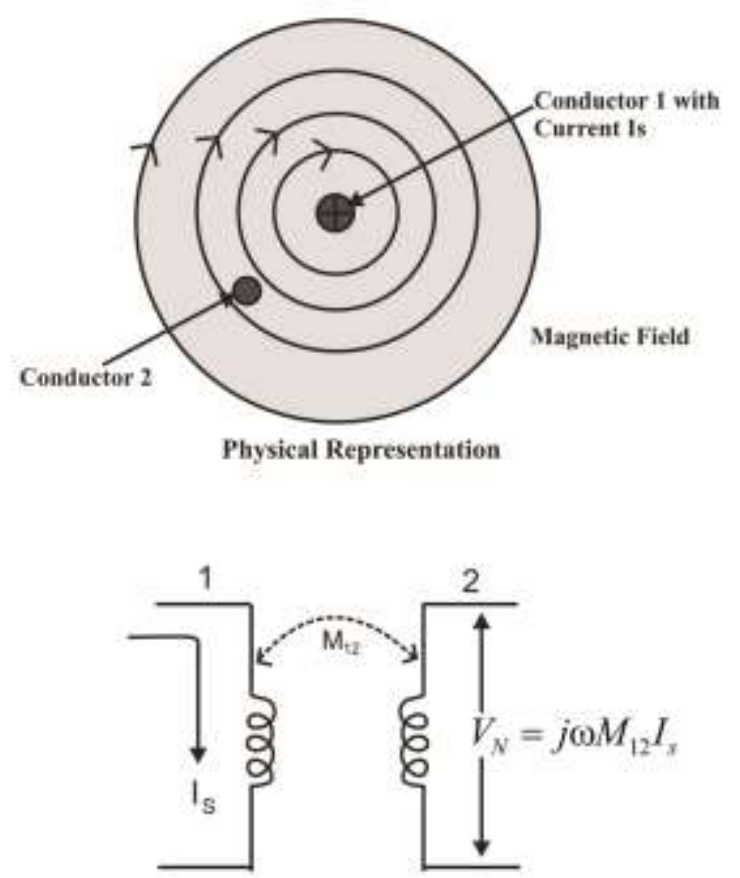

Equivalent Circuit

Fig. 4: When two circuits are coupled by a magnetic field, the coupling can be represented by a mutual inductance

\subsection{Coupling Mechanisms}

When cables are assumed short compared to a wavelength, the coupling mechanisms between circuits can be explained using lumped circuit components between circuits and analyzed using network theory ${ }^{[4]}$. The fig 5 shows the coupling mechanisms between two circuits, where power line is the source of interference and the receptor circuit, which is being affected by the interference is a networking cable.

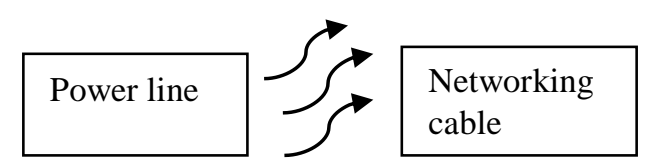

Fig 5: Coupling between two circuits

There are three types of coupling mechanisms ${ }^{[4,5]}$ :

1. Capacitive coupling or electric coupling: This type of coupling results from the interaction of electric fields between circuits.

2. Inductive coupling or magnetic coupling: This type of coupling results from the interaction between magnetic fields of two circuits.

3. Conductive Coupling: This type of coupling results from the common branch of two or more circuits.

Capacitive Coupling------The capacitive coupling between two conductors is represented in fig 6 using lumped circuit components. Let us consider that the AC voltage $\mathrm{V}_{1}$ on conductor 1 as the source of interference and conductor 2 as an UTP cabling which goes parallel. In the fig 6 , capacitance $C_{12}$ is the capacitance between conductors 1 and 2. Capacitance $\mathrm{C}_{1 \mathrm{G}}$ is the capacitance between conductor 1 and ground, capacitance $\mathrm{C}_{2 \mathrm{G}}$ is the total capacitance between conductor 2 and ground and $\mathrm{R}$ is the resistance of circuit 2 to ground. The equivalent circuit of the coupling is shown in fig 6 .
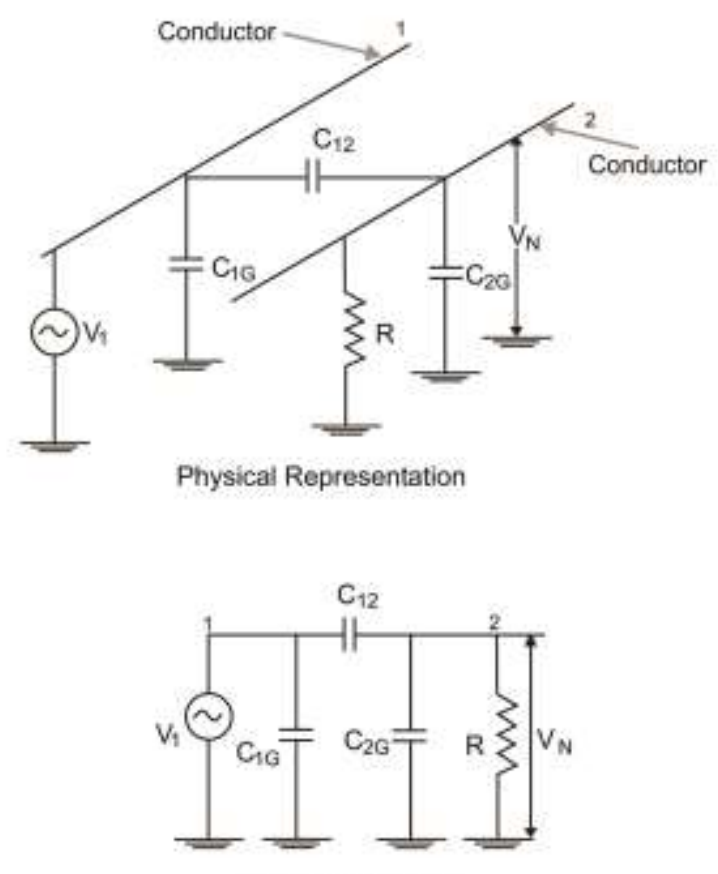

Equivalent Circuit

Fig. 6: Capacitive coupling between two conductors

The generated noise voltage $V_{N}$ produced between conductor 2 and ground is expressed as,

$$
\mathrm{V}_{\mathrm{N}}=\left[\mathrm{j} \omega\left(\mathrm{C}_{12} / \mathrm{C}_{12}+\mathrm{C}_{2 \mathrm{G}}\right) /\left\{\mathrm{j} \omega+1 / \mathrm{R}\left(\mathrm{C}_{12}+\mathrm{C}_{2 \mathrm{G}}\right)\right\}\right] \mathrm{V}_{1}
$$

When $\mathrm{R}<<1 / \mathrm{j} \omega\left(\mathrm{C}_{12}+\mathrm{C}_{2 \mathrm{G}}\right)$, the above equation can be reduced as the following:

$$
\mathrm{V}_{\mathrm{N}}=\mathrm{j} \omega \mathrm{RC}_{12} \mathrm{~V}_{1}
$$

Therefore, capacitive coupling or electric field coupling can be modeled as a current generator of magnitude $j \omega R C_{12} V_{1}$. This is shown in fig 7(a).

The equation (17) clearly shows that the produced noise voltage is directly proportional to the frequency $(\omega=2 n f)$ of the noise source, the resistance $\mathrm{R}$ of the affected circuit to ground, the capacitance $C_{12}$ between the conductors 1 and 2 and the voltage $V_{1}$ of the noise source. If we consider that the frequency and the voltage of the noise source are constant, the noise voltage is dependent upon the resistance $\mathrm{R}$ and the capacitance $\mathrm{C}_{12}$ between the conductors. In this case, noise voltage can be decreased by decreasing the resistance $R$ or decreasing the capacitance $C_{12}$. The 
value of the capacitance $\mathrm{C}_{12}$ between the two conductors can be decreased by physically separating the conductors, by shielding the conductors or by proper orientation of the conductors.

In the case, when $\mathrm{R}>1 / \mathrm{j} \omega\left(\mathrm{C}_{12}+\mathrm{C}_{2 \mathrm{G}}\right)$, the noise voltage,

$$
\mathrm{V}_{\mathrm{N}}=\left\{\mathrm{C}_{12} /\left(\mathrm{C}_{12}+\mathrm{C}_{2 \mathrm{G}}\right)\right\} \mathrm{V}_{1}
$$

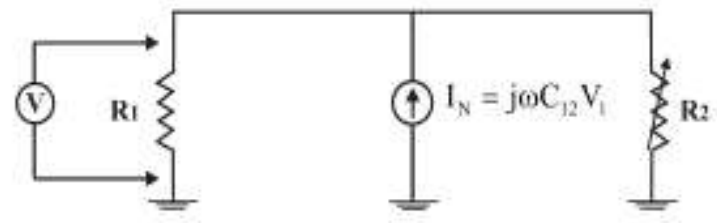

a) Electric Coupliag

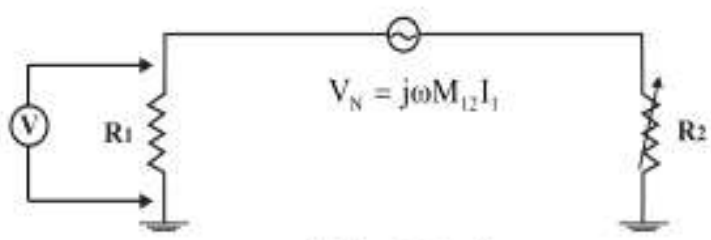

b) Magnetic Coupling

Fig. 7: (a) Equivalent circuit for electric field coupling, (b) Equivalent circuit for magnetic coupling

Inductive Coupling------When current flow in a circuit 1 produces a flux in another circuit 2 , there is a mutual inductance $M_{12}$ between the circuits 1 and 2 defined as

$$
\mathrm{M}_{12}=\varphi_{12} / \mathrm{I}_{1}
$$

Where, symbol $\varphi_{12}$ represents the flux in the circuit 2 due to the current $I_{1}$ in the circuit 1 .

Fig 8 shows the inductive coupling between two circuits. $\mathrm{I}_{1}$ is the current in the interfering circuit and $\mathrm{M}$ is the mutual inductance between the two circuits. Noise voltage $\mathrm{V}_{\mathrm{N}}$ can be expressed as,

$$
\mathrm{V}_{\mathrm{N}}=\mathrm{j} \omega \mathrm{MI}_{1}=\mathrm{MdI}_{1} / \mathrm{dt}
$$

There are some differences between magnetic and electric field coupling. For magnetic coupling, a noise voltage is generated in series with the receptor conductor (fig 7. b). But for electric field coupling, a noise current is produced between the receptor conductor and ground ${ }^{[3]}$ (fig 7. a).

Conductive Coupling------When two or more circuits have a common branch, conductive coupling occurs between them. If, circuit 1 is a power line and circuit 2 is a signal cable with a common ground system the parasitic current introduced into circuit 2 from circuit 1 results in an error (noise) voltage, which is harmful to the signals of circuit 2. When the bonding and grounding systems used for power and network cable system are sufficiently isolated, conductive coupling can never occur, otherwise conductive coupling is very common in cabling installations.
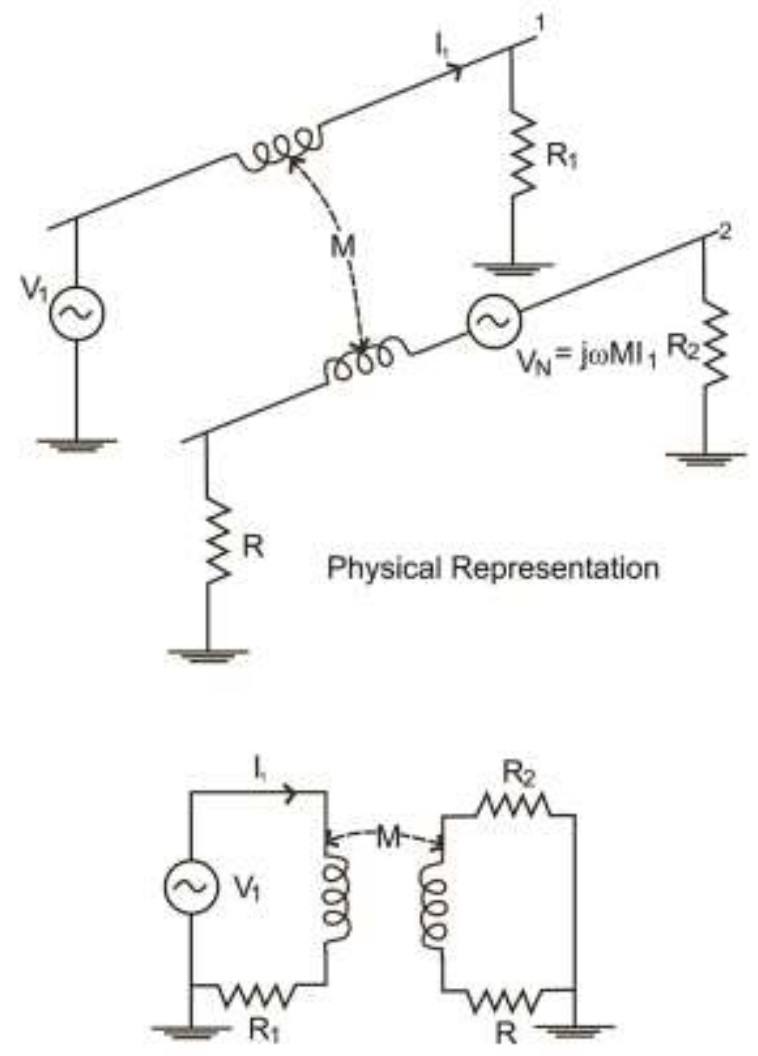

Equivalent Circuit

Fig. 8: Magnetic Coupling between two circuits

\section{Conclusion}

Electromagnetic Interference (EMI) is an undesirable phenomenon, which can degrade the performance of a system due to the electromagnetic fields. General Techniques used for controlling EMI are: Grounding, Shielding and Filtering. The performance of a network cabling system is depended upon the intensity of the EMI surrounding the system and how the system is protected against EMI. Electric noise usually cannot be eliminated, it can only be minimized to the point where it no longer causes interference. There are two effective methods to minimize the effect of EMI on UTP cablings: Physical Separation and Use of Shielding.

Electromagnetic Interference is caused by coupling mechanisms. There are two main coupling mechanisms, capacitive coupling and inductive coupling. Effect of an AC power line on UTP cable can be modeled and explained by using lumped circuit components $(\mathrm{R}, \mathrm{L}, \mathrm{C})$ and Network Theory. Distance between the source of interference and receptor plays an important role in controlling the effect of EMI. 


\section{References}

[1] G.Vijayaraghavan, Mark Brown, Malcolm Barnes, 'Grounding, Bonding, Shielding and Surge Protection', 2004.

[2] S.P. Seth, 'Elements of Electromagnetic Fields', Dhanpat Rai \& Co., $4^{\text {th }}$ edition 2011.

[3] Dipak L Sengupta, Valdis V. Liepa, 'Applied Electromagnetics and Electromagnetic Compatibility', John Wiley \& Sons, Inc, Hoboken New Jersey, 2006.

[4] Henry W. Ott., 'Noise Reduction Techniques in Electronic System', 2 ${ }^{\text {nd }}$ edition, Wiely, 1975.

[5] Network Cabling Solutions, 'Electromagnetic Interference', Technical Documents, The Siemon Company.

[6] S.N. Singh, 'Electric Power Generation, Transmission and Distribution', PHI, $8^{\text {th }}$ edition, 2007.

[7] C.L. Wadhwa, 'Electrical Power System', 2007.

[8] B.R. Gupta, 'Power System Design and Analysis' S. Chand, 2010.

[9] A. Chakrabarti, M.L. Soni, P.V. Gupta, U.S. Bhatnagar, 'Power System Engineering', Dhanpot Rai \& Co, 2008.

[10] B.M. Weedy, 'Electric Power System', $3^{\text {rd }}$ edition, 1987.

[11] Ashfaq Hussain, 'Electrical Power System', 5 ${ }^{\text {th }}$ edition, 2007.

[12] Vincent Del Toro, 'Electrical Engineering Fundamentals', PHI, $2^{\text {nd }}$ edition,2001

[13] Christos Christopoulos, 'Principles and Techniques of Electromagnetic Compatibility', CRC Press, Florida, 1995.

\section{Authors' Profiles}

Mitamoni Sarma: Assistant Professor and Head of Electronics in L.C.B. College of Guwahati, Assam, India. Post-graduated in Electronics and now working as a Ph.D. Scholar in Computer Science of Gauhati University. Her Research interest is in Communication and Circuits, and have already published four research papers on Power Line effect on UTP cables, and these are available in IEEE i-Explore.

Shikhar Kr. Sarma: Professor and Head of Information Technology in Gauhati University, Assam, India. His research Interest is in Communication Network Modeling, NLP and Expert Systems. He has produced 4 Ph.D. scholars and 8 scholars are currently working in his team. He has published more than 60 research papers in international journals and conference proceedings. He has also served in Program Committees and Technical Committees of many International Conferences.

How to cite this paper: Mitamoni Sarma, Shikhar Kr. Sarma,"An Analytical Study of Power Line Effect on UTP Cable using Lumped Circuit Components", International Journal of Information Technology and Computer Science(IJITCS), vol.5, no.12, pp.39-46, 2013. DOI: 10.5815/ijitcs.2013.12.05 